\title{
Treatment of haemobilia by selective arterial embolisation
}

\author{
E A FAGAN,* D J Allison, V S CHADWICK, AND H J F HODGSON \\ From the Departments of Medicine and Radiology, Royal Postgraduate Medical School, \\ Hammersmith Hospital, London
}

SUMMARY We report a patient in whom haemobilia occurred after percutaneous liver biopsy. Selective hepatic arteriography showed a fistula between hepatic artery and portal venous system, with appearance of contrast in the biliary tract. Intrahepatic bleeding was stopped by arterial embolisation with a mixture of gelatine foam and sterile dura mater. Cholecystectomy was subsequently required as a haemocholecyst developed. The technique of arteriography and embolisation allows accurate localisation of intrahepatic bleeding sites and may avoid the need for a direct surgical approach to this problem.

In a prospective study, $7 \%$ of patients undergoing percutaneous liver biopsy developed scintigraphic evidence of intrahepatic haematomas. ${ }^{1}$ Significant haemobilia, haemorrhage into the biliary tree, is however, extremely rare after this procedure. Between 1967 and 1977, 13 patients with haemobilia were reported in the literature. ${ }^{2-11}$ Three of those patients died. A variety of management approaches have been used, ranging from aggressive surgery to conservative management. We report a patient treated by arterial embolisation during hepatic angiography and advocate this approach.

\section{Case report}

A 45 year old Pakistani woman was admitted with a six day history of jaundice, pain in the right upper quadrant, fever, dysuria, and rash. She had taken dihydrocodeine, sulindac, and cotrimoxazole.

She was a thin, icteric woman without stigmata of chronic liver disease. Abdominal examination revealed tender hepatomegaly and the spleen was just palpable.

The haemoglobin was $12.6 \mathrm{~g} / \mathrm{dl}$ and leucocyte count, platelets and coagulation screen were normal. Serum bilirubin was $200 \mu M / 1 \quad(n<14)$. Aspartate amino transferase $246 \mathrm{IU} / \mathrm{l}(\mathrm{n}<40)$ and

\footnotetext{
*Address for correspondence and reprints: Dr E A Fagan, Gastroenterology Unit, RPMS, Hammersmith Hospital, Ducane Road, London W2 0HS.
}

Received for publ: sation 4 December 1979 alkaline phosphatase $595 \mathrm{IU} / 1 \quad(\mathrm{n}<133)$. Hepatic ultrasound and scintiscan were normal.

In view of persisting fevers to $40^{\circ} \mathrm{C}$ and a rising serum alkaline phosphatase, a percutaneous liver biopsy was performed 14 days after admission, via the lateral approach, using a Tru-cut needle (Travenol-New Jersey). The histological appearance of the biopsy was that of a drug-induced hepatitis.

Forty-eight hours later the patient experienced three episodes of colicky, right upper quadrant pain. Two days later fresh melaena was detected on rectal examination and the haemoglobin had fallen to $9.4 \mathrm{~g} / \mathrm{dl}$. Endoscopy showed altered blood in the stomach with fresh blood issuing from the ampulla of Vater. Over the ensuing 48 hours melaena continued and six units of blood were transfused.

Six days after biopsy, angiography was performed to locate the bleeding site and attempt its embolisation. Selective hepatic arteriography was performed via the femoral route, using a steerable catheter (Muller, US Catheter International Inc., New Jersey). Radiographs early in the arterial phase demonstrated an arteriovenous fistula (Fig. 1), with early filling of the portal venous tree. Late films showed contrast within the common bile duct (Fig. 2). Selective embolisation of the branch of the right hepatic artery supplying the abnormal segment was performed using sterile, absorbable, gelatine sponge (Sterispon, Allen and Hanbury, London) with sterile human dura mater (Lyodura, Davis and Geck, Cynamid, Gosport, Hants). 


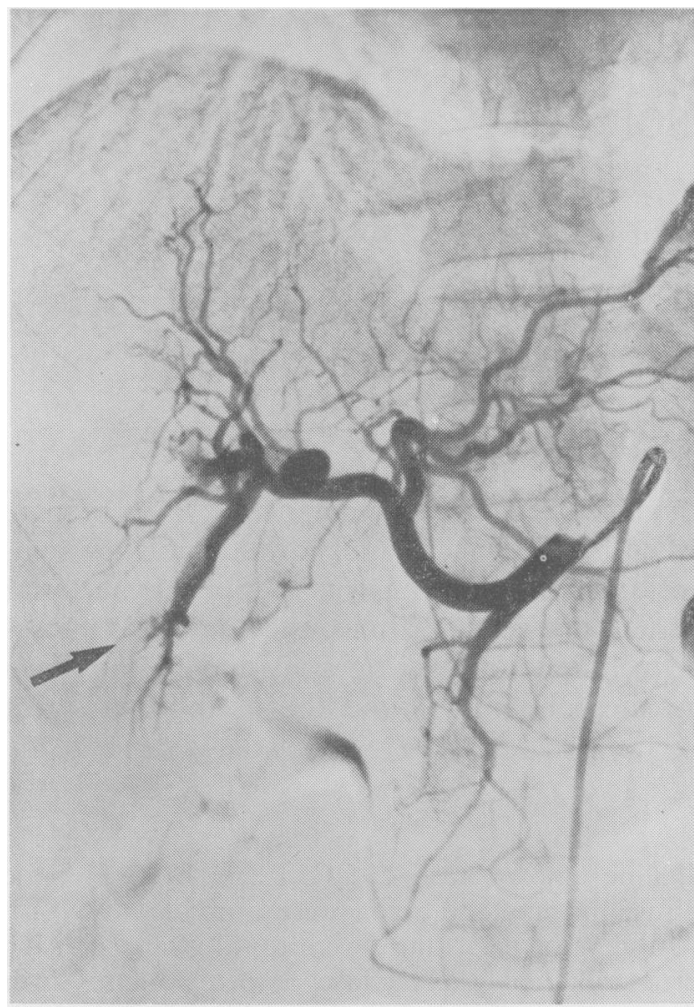

Fig. 1 Selective hepatic arteriogram. Early arterial phase with early portal vein filling at site of fistula (arrow).

Angiography at the end of this procedure confirmed obliteration of the vascular supply to the abnormal segment, while the remainder of the liver was unaffected (Fig. 3). After the procedure, the patient's pain ceased, with no further evidence of bleeding.

Two weeks after embolisation, the patient complained of a new constant pain over the liver. She was well and apyrexial with minimal jaundice but a firm, mobile, and tender gallbladder was now palpable. Ultrasound showed an enlarged, thickened gallbladder with echogenic areas interpreted as 'stones and sludge', a dilated cystic duct but normal bile ducts. Repeat gastroscopy and scintiscan were normal.

A laparotomy was performed. The gallbladder was oedematous, full of blood clot and clot was evacuated from the cystic and common bile ducts. The cystic artery was pulsatile and the blood supply to the gallbladder had not been compromised by the embolisation procedure. A cholecystectomy was performed and T-tube cholangiogram 14 days postoperatively was normal. Six months later the patient remains well with normal haemoglobin and liver function tests.

\section{Discussion}

In this particular patient, haemobilia after percutaneous liver biopsy was not a diagnostic problem; she presented the classical symptom complex of biliary colic, jaundice, and gastrointestinal haemorrhage, and endoscopy confirmed the diagnosis by showing fresh blood emerging from the ampulla of Vater. The management of this complication, however, remains difficult. In three of the 13 patients reported in the literature, bleeding ceased spontaneously after a period of conservative management with transfusion alone ${ }^{458}$ but one fatality has followed this approach. ${ }^{10}$ Bleeding may also recur after a period of some weeks. ${ }^{8}$ Experience with haemobilia after blunt trauma to the abdomen has encouraged a policy of early surgical intervention ${ }^{12}$ and some patients have therefore been treated with major procedures including hemi-hepatectomy and hepatic artery ligation ${ }^{4}$; these procedures have a significant mortality of their own, particularly in

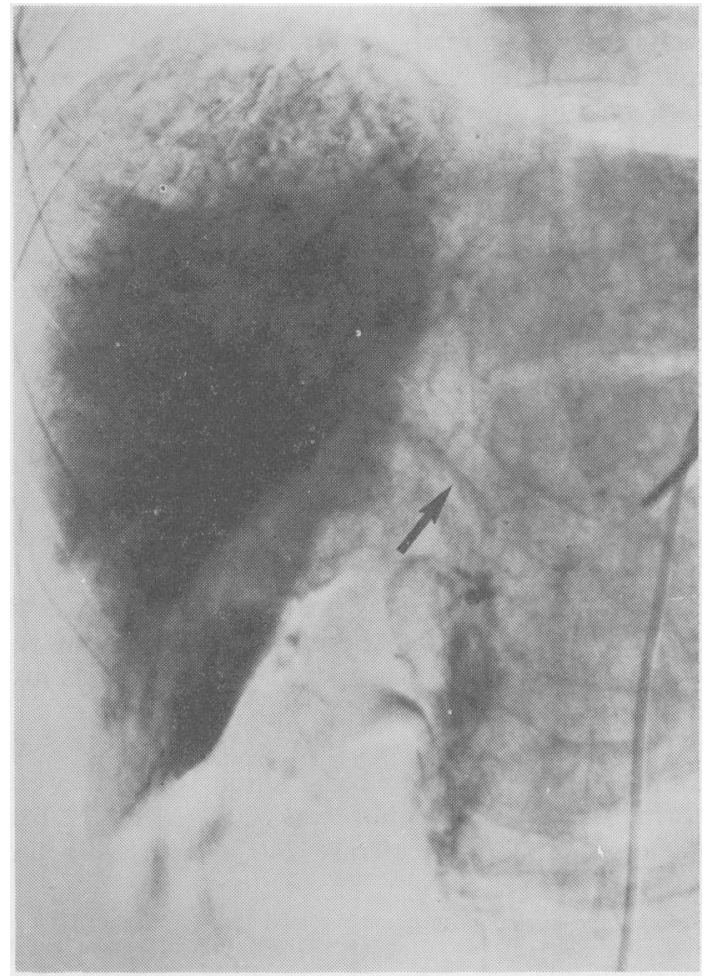

Fig. 2 Selective hepatic arteriogram. Late phase showing contrast medium in common bile duct (arrow). 
patients with diffuse parenchymal liver disease. Two of the six patients undergoing such surgical procedures for control of haemobilia died. ${ }^{4}$ Obviously no conclusions can be drawn from comparisons between such small numbers of patients, and assessment of the results of different forms of therapy may well be made more complicated by bias against reporting fatalities.

The direct arteriographic approach used here has a number of advantages. In the 10 patients in whom it has been used the bleeding site has been accurately located in all but one. ${ }^{6}$ This is of particular advantage if gastrointestinal haemorrhage after liver biopsy is not associated with either biliary colic or definite findings at endoscopy. Furthermore, definitive therapy can be instituted. One possible approach was used by Lee et al., who infused adrenaline and propranolol into the right hepatic artery. After a half-hour infusion and after severe pain had developed, bleeding stopped. The other approach is that of direct embolisation of the bleeding site.

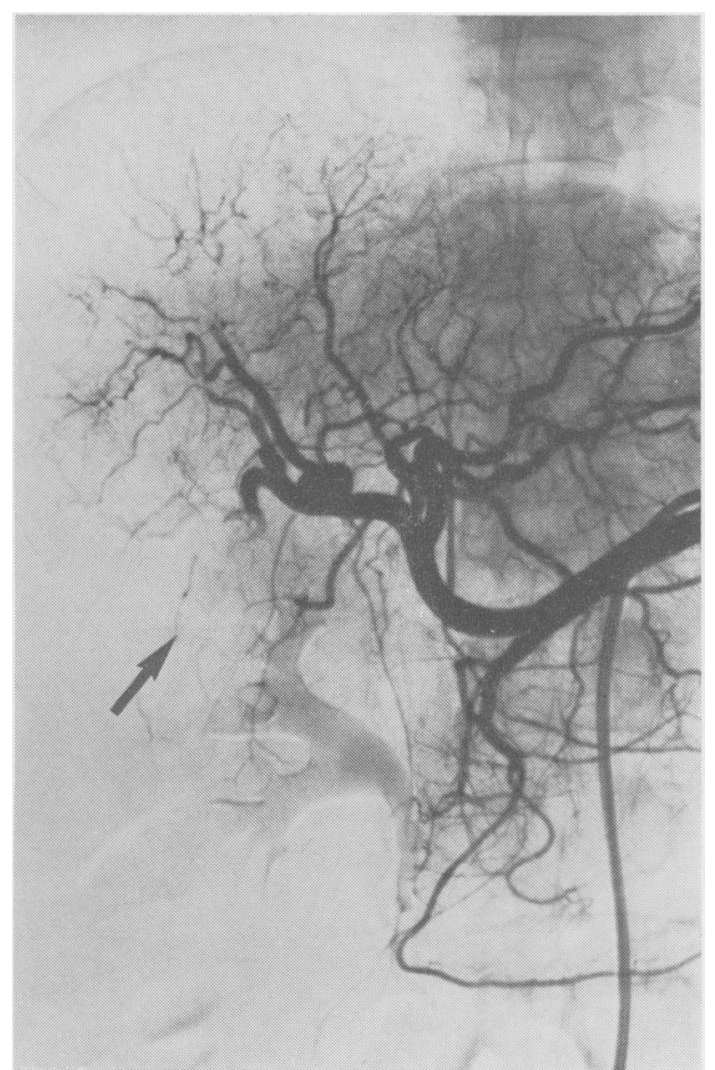

Fig. 3 Selective hepatic arteriogram, after embolisation. Segment containing site of arteriovenous fistula fails to opacify (arrow).
This has been used in two previous cases of post-liver biopsy haemobilia, ${ }^{911}$ and has also been used for the treatment of haemobilia after blunt trauma to the liver. ${ }^{13} 14$ Direct embolisation of the arteriovenousbiliary fistula in our patient led to the immediate relief of pain and cessation of haemorrhage, the latter confirmed angiographically. Highly selective embolisation was possible as a steerable catheter could be positioned sufficiently close to the bleeding site. It is clearly desirable that only a small proportion of the liver be deprived of its arterial blood supply, particularly in the presence of generalised liver disease; even when such a selective approach is not possible, emboli tend to stream in towards areas of high blood flow and thus are likely to obliterate arteriovenous fistulae at biopsy sites. Emboli consisting of gelatine foam with collagen were used, as there is evidence that this combination produces permanent closure of vascular anomalies. ${ }^{15}$ In a case of haemobilia after blunt trauma treated with embolisation by gel foam alone, recurrence of a false aneurysm was documented and repeat embolisation was required.$^{14} \mathrm{~A}$ further advantage of arteriography is that accurate localisation of the bleeding site and demonstration of the hepatic vascular anatomy provides valuable information for the surgeon should embolisation prove impossible or unsuccessful.

In the patient treated here, although haemorrhage was successfully arrested by arteriographic embolisation, surgery was eventually required for a haemocholecyst, a well-described complication of haemobilia. The procedure required to remove the blood clot from the biliary tree was considerably less hazardous than a direct approach to the bleeding site. Similarly, in the first patient treated with this procedure, by Walters et al., haemorrhage was successfully arrested after embolisation of a bleeding arteriovenous fistula. Subsequent evacuation of a large intrahepatic haematoma was necessary, however, to relieve pain. We do not know whether embolisation earlier in these two patients would have prevented the development of these complications. De Villasante and his colleagues successfully embolised a bleeding hepatic artery 14 days after biopsy and surgery was not required.

In patients who are continuing to bleed into tre biliary tree after percutaneous liver biopsy, direct embolisation of the biopsy site at arteriography appears to offer a safe and effective means of treatment.

\section{References}

\footnotetext{
${ }^{1}$ Raines DR, Van Heertum RL, Johnson LF. Intrahepatic hematoma: a complication of percutaneous liver biopsy. Gastroenterology 1979; 67: 284-9.
} 
${ }^{2}$ Cox EF. Hemobilia following percutaneous needle biopsy of liver. Arch Surg 1967; 95: 198-201.

${ }^{3}$ Berg D. Two complications following needle biopsy of the liver. Pakistan Surg Gynecol Obstet 1970; 12: 745-9.

${ }^{4}$ Levinson JD, Olsen G, Terman JW, Cleveland CR, Graham CP Jr, Breen KJ. Hemobilia secondary to percutaneous liver biopsy. Arch Intern Med 1972; 130: 396-400.

${ }^{5}$ Sandblom P. Haemobilia (biliary tract hemorrhage): history, pathology, diagnosis, treatment. Springfield, Ill: Thomas, 1972.

${ }^{6}$ Ball TJ, Mutchnik MG, Cohen GM, Burrell M. Hemobilia following percutaneous liver biopsy. Gastroenterology 1975; 68: 1297-9.

${ }^{7}$ Attiyeh, FF, McSweeney J, Fortner JG. Haemobilia complicating needle liver biopsy. Radiology 1976; 118: $559-60$.

${ }^{8}$ Seltzer RA, Rossiter SB, Cooperman LR, Liebowitz D. Hemobilia following needle biopsy of the liver. $A J R$ 1976; 127: 1035-6.
${ }^{9}$ Walter JF, Paaso BT, Cannon WB. Successful transcatheter embolic control of massive haematobilia secondary to liver biopsy. $A J R$ 1976; 127: 847-9.

${ }^{10}$ Lee SP, Tasman-Jones C, Wattie WJ. Traumatic hemobilia: a complication of percutaneous liver biopsy. Gastroenterology 1977; 72: 941-4.

${ }^{11}$ Merino-de Villasante J, Alvarez-Rodriguez RE, Hernandez-Ortiz J. Management of postbiopsy hemobilia with selective arterial embolization. AJR 1977; 128: 668-71.

${ }^{12}$ Whelan TJ, Gillespie JT. Treatment of traumatic hemobilia. Ann Surg 1965; 162: 920-32.

${ }^{13}$ Bass EM, Crosier JH. Percutaneous control of posttraumatic hepatic hemorrhage by gelfoam embolization. J Trauma 1977; 17: 61-3.

${ }^{14}$ Heimbach DM, Ferguson GS, Harley JD. Treatment of traumatic haemobilia with angiographic embolization J Trauma 1978; 18: 221-4.

${ }^{15}$ Allison DJ. Therapeutic embolization. $\mathrm{Br} J$ Hosp Med 1978; 20 : 707-14. 